\title{
CREMONA TRANSFORMATIONS WITH AN INVARIANT RATIONAL SEXTIC
}

\author{
A. B. COBLE
}

It is well known that a Cremona transformation $T$ with ten or fewer $F$-points will transform a rational sextic $S$ into a rational sextic $S^{\prime}$ when the $F$-points of $T$ are all located at the nodes of $S$. I have shown (cf. [1], p. 248, (9); [2], p. 255, (5)) that, even though the number of transformations $T$ of the type indicated is infinite, the transforms $S^{\prime}$ are all included in $2^{13} \cdot 31 \cdot 51$ classes, the members of any one class being all projectively equivalent and a member of one class being projectively distinct from a member of another class. The sextic $S$ itself is in one of these classes, $T$ being then the identity. If $S^{\prime}$ is in the same class as $S$, and if $C$ is the collineation which carries $S^{\prime}$ into $S$, then $T C$ is a Cremona transformation of the same type as $T$ which transforms $S$ into itself. There is thus an infinite group of Cremona transformations which carry $S$ into itself. If $t$ is a parameter on $S$, the effect of an element of such a group on the points of $S$ is represented by

$$
t^{\prime}=\frac{a t+b}{c t+d}, \quad \quad a d-b c \neq 0 .
$$

It is an obvious question as to whether transformations $T$, other than the identical collineation, exist for which (1) reduces to $t^{\prime}=t$; that is, whether $S$ can be a locus of fixed points of a transformation $T$. I had expressed the opinion that such transformations $T$ do not exist (cf. [1], end of $\$ 3$ ). It was therefore most interesting to find in a recent article of G. Pompili (Pompili [1]) a purported construction of such a transformation. However the examination, made in the following, of this transformation shows that the construction is fallacious.

Let $S$ be a generic rational sextic with nodes at $p_{1}, \cdots, p_{7}$ and at $A, B, C$. Let $H$ be a generic member of the pencil $(H)$ of elliptic sextics with nodes at $p_{1}, \cdots, p_{7}, B, C$, the pencil being determined by $S$ and the square of the cubic $\left(p_{1} \cdots p_{7} B C\right)^{3}$. On $H$ let $g_{B}, g_{C}$ denote the pairs of points at the nodes. Then on this elliptic curve the equivalence

$$
T: P^{\prime}-P \equiv g_{B}-g_{C}
$$

determines a birational correspondence which, extended over the various members of the pencil $(H)$, yields a Cremona transformation $T$ of the plane. If $\left(H^{\prime}\right),\left(H^{\prime \prime}\right)$ are similar pencils 


$$
\left(p_{1}{ }^{2} \cdots p_{7}{ }^{2} C^{2} A^{2}\right)^{6}, \quad\left(p_{1}{ }^{2} \cdots p_{7}{ }^{2} A^{2} B^{2}\right)^{6},
$$

respectively, and if on $H^{\prime}$ and $H^{\prime \prime}$, respectively, the pairs of nodes $C, A$ and $A, B$ are represented by $g_{C^{\prime}}, g_{A}{ }^{\prime}$ and $g_{A}{ }^{\prime \prime}, g_{B}{ }^{\prime \prime}$, then similar Cremona transformations $T^{\prime}$ and $T^{\prime \prime}$ exist which are determined by the equivalences

$$
T^{\prime}: P^{\prime}-P \equiv g_{C}{ }^{\prime}-g_{A}{ }^{\prime}, \quad T^{\prime \prime}: P^{\prime}-P \equiv g_{A}{ }^{\prime \prime}-g_{B}{ }^{\prime \prime} .
$$

The three pencils $(H),\left(H^{\prime}\right),\left(H^{\prime \prime}\right)$ have one common member $S$.

Pompili remarks that on $S$ we have the equivalences

$$
g_{B} \equiv g_{A}{ }^{\prime}, \quad g_{C} \equiv g_{A}{ }^{\prime \prime}, \quad g_{C}{ }^{\prime} \equiv g_{B}{ }^{\prime \prime},
$$

whence it is "intuitive" that the transformation $R=T^{\prime \prime} T^{\prime} T$ has $S$ as a locus of fixed points. The fallacy seems to rest in the definition of $T$ in (2). This definition is precise for every elliptic member of $(H)$, but it becomes illusory for each of the twelve rational members of $(H)$ including $S$. For, on a rational curve, we have $P^{\prime} \equiv P$ and $g_{B} \equiv g_{C}$, and $T$ in (2) takes the form $0 \equiv 0$. Even the conclusion that $R$ has on $S$ the expression $P^{\prime}-P \equiv 0$ would give on $S$ no information as to the relative position of $P$ and $P^{\prime}$.

The nature of the Cremona transformation $R$ may be determined in the following way which also shows that $S$ is not a locus of fixed points of $R$. Let $b_{A}, b_{B}, b_{C}$, be the three Bertini involutions with seven $F$-points at $p_{1}, \cdots, p_{7}$ and with their eighth $F$-points at $A, B$, $C$, respectively. By definition, $b_{B}$ carries every 8-nodal sextic $\left(p_{1}^{2} \cdots p_{7}^{2} B^{2}\right)^{6}$ into itself and therefore carries every cubic of the pencil $\left(p_{1} \cdots p_{7} B\right)^{3}$ into itself. Thus the pairs of the involution $b_{B}$ on each such 8-nodal sextic are cut out by the members of this pencil of cubics. In particular, $H$ is such an 8-nodal sextic and $g_{C}$ is one of the pairs of $b_{B}$. Thus the parametric expression of $b_{B}$ on $H$ with pairs $P P^{\prime \prime}$ is $P+P^{\prime \prime} \equiv g_{C}$. Similarly the expression on $H$ of $b_{C}$ with pairs $P^{\prime} P^{\prime \prime}$ is $P^{\prime}+P^{\prime \prime} \equiv g_{B}$. Hence $P^{\prime}-P \equiv g_{B}-g_{C}$. Thus the transformation $T$ in (2) which carries $P$ into $P^{\prime}$ is $b_{B} b_{C}$. Hence the transformations $T, T^{\prime}, T^{\prime \prime}, R$ used above are expressible in terms of Bertini involutions as follows:

$$
T=b_{B} b_{C}, \quad T^{\prime}=b_{C} b_{A}, \quad T^{\prime \prime}=b_{A} b_{B}, \quad R=b_{A} b_{B} \cdot b_{C} b_{A} \cdot b_{B} b_{C} .
$$

It is easy to obtain the parametric equation on $S$ of the transformation $R$. Let the nodal parameters of $S$ at $A, B, C$, respectively, be the pairs $(a t)^{2},(b t)^{2},(c t)^{2}$, and let the jacobians of these pairs be

$$
(\alpha t)^{2}=J(b, c), \quad(\beta t)^{2}=J(c, a), \quad(\gamma t)^{2}=J(a, b) .
$$


Let also $r_{\alpha}, r_{\beta}, r_{\gamma}$ be the binary involutions with fixed points $(\alpha t)^{2}$, $(\beta t)^{2},(\gamma t)^{2}$, respectively. Then the Bertini involution $b_{A}$ has $B$ and $C$ for fixed points and interchanges the nodal directions at each. Hence its effect on $S$ is given by $r_{\alpha}$. Thus the parametric equation on $S$ of the transformation $R$ is

$$
R=r_{\alpha} r_{\beta} \cdot r_{\gamma} r_{\alpha} \cdot r_{\beta} r_{\gamma} .
$$

Since $S$ is generic, the quadratics $(a t)^{2},(b t)^{2},(c t)^{2}$ are generic, and their three jacobians $(\alpha t)^{2},(\beta t)^{2},(\gamma t)^{2}$ are likewise three generic quadratics. Then $R \neq 1$; that is, $S$ is not a locus of fixed points. For, if $R=1$, there exists a binary involution $r_{\delta}=r_{\alpha} r_{\beta} r_{\gamma}$ such that $r_{\alpha} r_{\beta} r_{\gamma} r_{\delta}=1$. This would require that the jacobians of $(\alpha t)^{2},(\beta t)^{2}$ and of $(\gamma t)^{2},(\delta t)^{2}$ should coincide; that is, $(\alpha t)^{2},(\beta t)^{2},(\gamma t)^{2}$ would have a common apolar quadratic, and would therefore lie in a pencil and thus be non-generic.

Pompili ([1], p. 85) sets up the three transformations $r_{\alpha} r_{\beta}, r_{\gamma} r_{\alpha}$, $r_{\beta} r_{\gamma}$ and multiplies them together to get the result $R=1$. However, he uses the following order of multiplication, $r_{\beta} r_{\gamma} \cdot r_{\gamma} r_{\alpha} \cdot r_{\alpha} r_{\beta}$, which obviously yields the identity. If this is his definition of $R$, the corresponding Cremona transformation $R$ is $b_{B} b_{C} \cdot b_{C} b_{A} \cdot b_{A} b_{B}$ which is, just as obviously, the ternary identical collineation rather than a Cremona transformation.

It may be observed that the Cremona transformation $R$ defined in (5) has the order 2305. It has $p_{1}, \cdots, p_{7}$ as $F$-points of order 768 and $A, B, C$ as $F$-points of respective orders $1008,384,144$. The numerical matrix which describes its direct and inverse $F$-points and $P$-curves reads as follows:

\begin{tabular}{c|cccccccc} 
& & $p_{1}$ & $p_{2}$ & $\cdots$ & $p_{7}$ & $A$ & $B$ & $C$ \\
\hline & 2305 & -768 & -768 & $\cdots$ & -768 & -1008 & -384 & -144 \\
$p_{1}$ & 768 & -255 & -256 & $\cdots$ & -256 & -336 & -128 & -48 \\
$p_{2}$ & 768 & -256 & -255 & $\cdots$ & -256 & -336 & -128 & -48 \\
$\cdot$ & $\cdot$ & $\cdot$ & $\cdot$ & $\cdots$ & $\cdot$ & $\cdot$ & $\cdot$ & $\cdot$ \\
$p_{7}$ & 768 & -256 & -256 & $\cdots$ & -256 & -336 & -128 & -48 \\
$A$ & 144 & -48 & -48 & $\cdots$ & -48 & -63 & -24 & -8 \\
$B$ & 384 & -128 & -128 & $\cdots$ & -128 & -168 & -63 & -24 \\
$C$ & 1008 & -336 & -336 & $\cdots$ & -336 & -440 & -168 & -63
\end{tabular}

The first column of this table indicates that $R$ transforms lines into curves of order 2305 with points of order 768 at $p_{1}, \cdots, p_{7}$ and with points of orders $144,384,1008$ at $A, B, C$, respectively; the second column, that $R$ transforms the set of directions at $p_{1}$ into a curve of order 768 with points of order 255 at $p_{1}$, of order 256 at 
$p_{2}, \cdots, p_{7}$, and of orders 48,128 , and 336 at $A, B$, and $C$, respectively; and so on.

\section{REFERENCES}

A. B. Coble

1. The ten nodes of the rational sextic and the Cayley symmetroid, American Journal of Mathematics, vol. 41 (1919), pp. 243-265.

2. Algebraic Geometry and Theta Functions, American Mathematical Society Colloquium Publications, vol. 10, New York, 1929.

Giuse pre Pompili

1. Sulle trasformazioni cremoniane del piano che posseggono una curva di punti uniti, Rendiconti del Seminario Matematico della Università di Roma, (4), vol. 2 (1938), pp. 47-87.

UNIVERSITY OF ILLINOIS

\section{A NOTE ON THE REDUCTION OF GENTZEN'S CALCULUS LJ*}

H. B. CURRY

The reduction given by Gentzen $\dagger$ of his calculus $\mathrm{LJ}$ to the modified Heyting calculus LHJ is somewhat involved because he reduces everything to the axioms without assuming any knowledge of the calculus. By the use, however, of certain general theorems it is possible to simplify the reduction. The purpose of this note is to present an alternative reduction based on this principle. Although this new reduction may, if all the assumptions used are proved in detail from the axioms, conceivably be longer than Gentzen's, yet the formulas and principles established at the beginning (in $\$ \$ 1-4$ below) are for the most part well known (or at least of some interest on their own account), and in terms of these the reduction (in §5) is almost immediate.

The new method has the further merit of showing, if we take the axioms of LHJ as a basis, that the schemes for implication follow from the axioms for implication only $\ddagger$ and that those for conjunction, negation, and the quantifiers, respectively, involve only the axioms for implication and those for the operation concerned. $\$$ It

* Presented at a joint meeting of the Society and the Association for Symbolic Logic, December 29, 1937.

$\dagger$ G. Gentzen, Untersuchungen über das logische Schliessen, Mathematische Zeitschrift, vol. 39 (1934), pp. 417-428.

¥ The scheme "Verdünnung im Sukzedens" is essentially a scheme for negation.

$\S$ This statement requires that the formula 1.42 (below) be postulated as axiom of $\mathrm{LHJ}$; to derive 1.42 from $\mathrm{LHJ}$ as it stands requires properties of conjunction. 